\title{
Anisotropic Properties of Sculptured Thin Films
}

\author{
Drew Pulsifer, Andrew Jones and Tariq H. Gilani \\ Department of Physics \\ Millersville University \\ P. O. Box 1002 \\ Millersville, Pennsylvania 17551 USA
}

Received: October 14, 2008

Accepted: January 10, 2009

\begin{abstract}
The electrical anisotropy of Cr-Sculptured thin films has been studies as a function of growth angle of $\mathrm{Cr}$ columns on a glass substrate. A peak in resistivity anisotropy is found at growth angle of $72 \pm 5^{\circ}$.

\section{INTRODUCTION}

Sculptured Thin Films (STFs) are essentially nano-engineered thin films [1] comprising on columns of parallel, curvilinear shaped nanowires [2 - 6]. With advancements in technology [1,2], it is now possible to grow these columns from any material (insulators, semiconductors or metals) and with desired architect on a substrate. This manipulation of the material on nano scale results in interesting optical and other the properties of STFs [1 - 5] and as a result numerous applications of STFS have been suggested [1 - 8].

If a STF is prepared from a metal on an insulating substrate, its electrical properties may also be varied by varying its architect. In this study we investigated the

anisotropy in electrical conductivity of the Chromium STFs as a function of columns growth angle.

\section{EXPERIMENTAL}

The samples of Sculptured Thin Films were obtained from the Department of Engineering Sciences and Mechanical Engineering, Pennsylvania State University [9]. These samples were made from Chromium columns grown on glass substrate at various angles ranging from 0 to 85 degrees with normal to substrate. Montgomery method [10] was applied to find the ratios of electrical resistivities in $X$ - and $Y$ - directions. These directions are shown in Figure 1(a). The electrical conductivity in Zdirection (normal to substrate) is assumed to
\end{abstract}

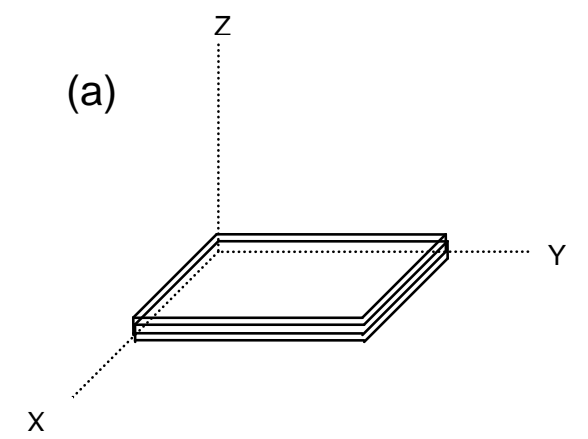

(b)

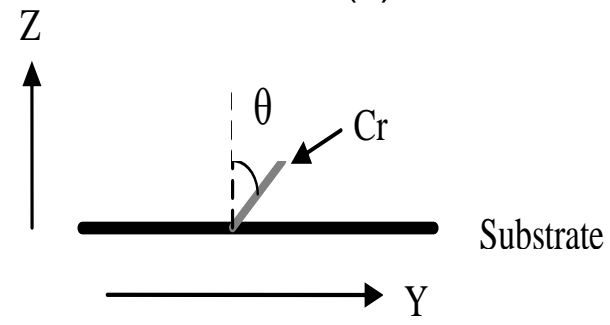

Figure 1. Schematic diagram of (a) $\mathrm{X}$ - and $\mathrm{Y}$-directions and (b) Angle $\theta$ of Chromium columns with normal to substrate. 


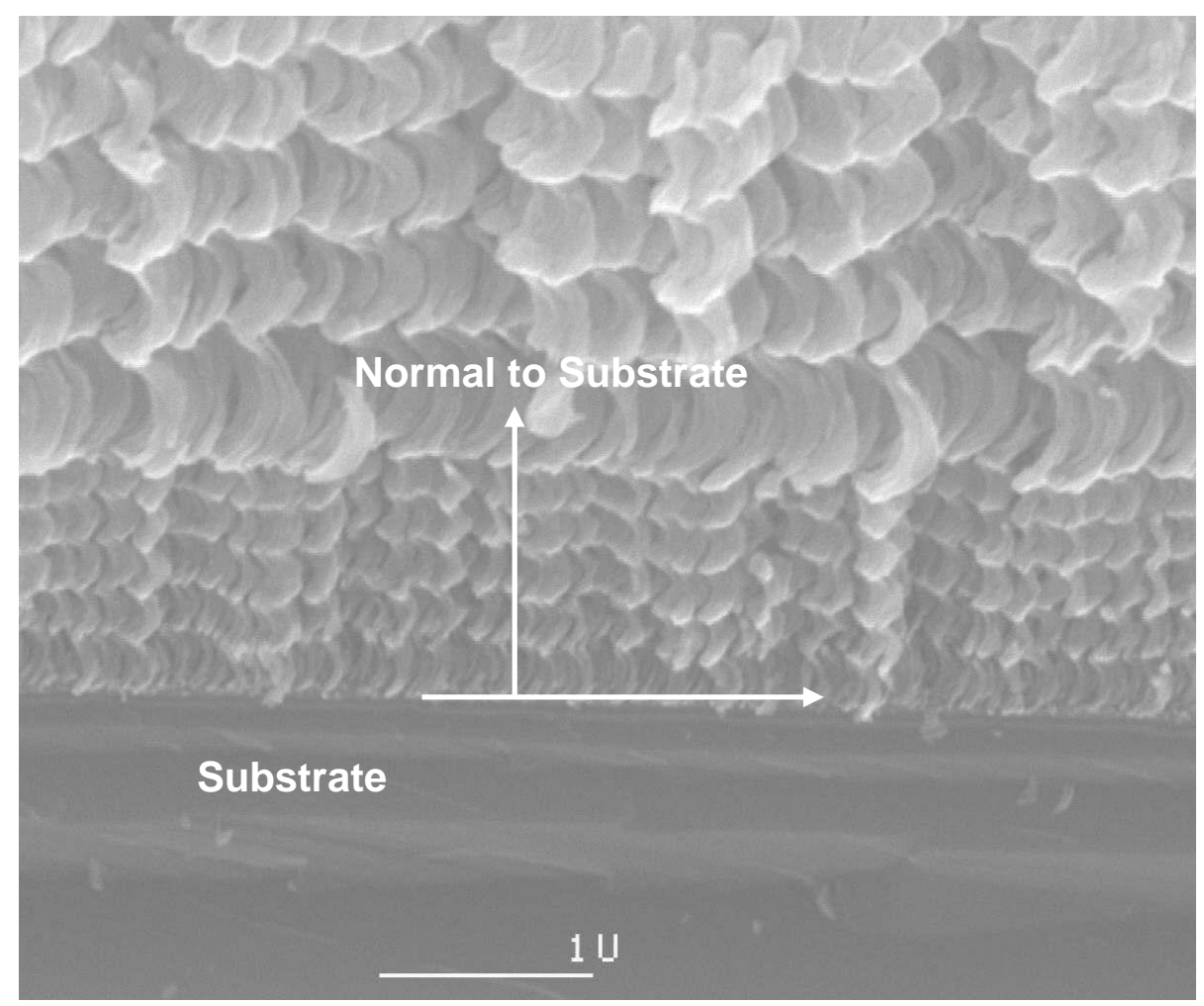

Figure 2. STM monograms of a typical sample. Note that only for $\theta=0^{\circ}$ is shown in the picture.

be very high as compared with conduction in $X$ - or $Y$-direction. The angle $\theta$ measured from normal to substrate and is varied $(0$ to 85 degrees) in $\mathrm{Y}-\mathrm{Z}$ plane as schematically shown in Fig 1 (b).

Figure 2 depicts the STM monograms of a typical sample with $\mathrm{Cr}$ Columns growth angle of zero degree. All the samples were baked at $120{ }^{\circ} \mathrm{C}$ for several hours before the measurements to avoid any moisture trapped among the columns. All the measurements were made in a moisture free environment at room temperature. Kiethley model 2400 source meter and Keithley model 2001 multimeter were used as controlled dc current source and for the measurement of small voltages, respectively. Small current was required to avoid any thermal effects. A typical I-V curve is shown in Figure 3.

\section{RESULTS AND DISCUSSION}

Resistivity ration $\left(\rho_{x} / \rho_{y}\right)$ as a function of column angle $\theta$ is shown in Fig 4 , where $\rho_{x}$ and $\rho_{y}$ are the room temperature electrical resistivities in $\mathrm{X}$ - and $\mathrm{Y}$-directions respectively. Clearly the anisotropy increases exponentially as $\theta$ increases until it reaches to its maximum value and then drops exponentially, as shown in Figure 4 (bottom). The maximum anisotropy and angle $\theta$ at which maximum anisotropy results are found by plotting the data on semi log scale and extrapolating the data from both sides as shown in Figure 4 (top). The value of maximum anisotropy is 5.8 and the $\theta$ for maximum anisotropy is $72 \pm 5^{\circ}$.

We believe that the resistivity anisotropy in Cr-STFs is due to the inherent structural inhomogenieties of these films [1, 11]. As columns grow in height, they also grow in diameter [7] and hence the intercolumnar distance decreases. When the growth angle is large, the shadowing stops the column growth in vapor flux direction $(Y$ direction in this case), while columns keep on growing in transverse directions ( $X$ - and $Z$-direction) resulting in further decrease in inter columnar distance along the X-direction [2] and hence decrease in resistivity $\rho_{x}$. Therefore, first $\rho_{x} / \rho_{y}$ increases as $\theta$ increases and then starts decreasing after reaching to a maximum value at a certain 


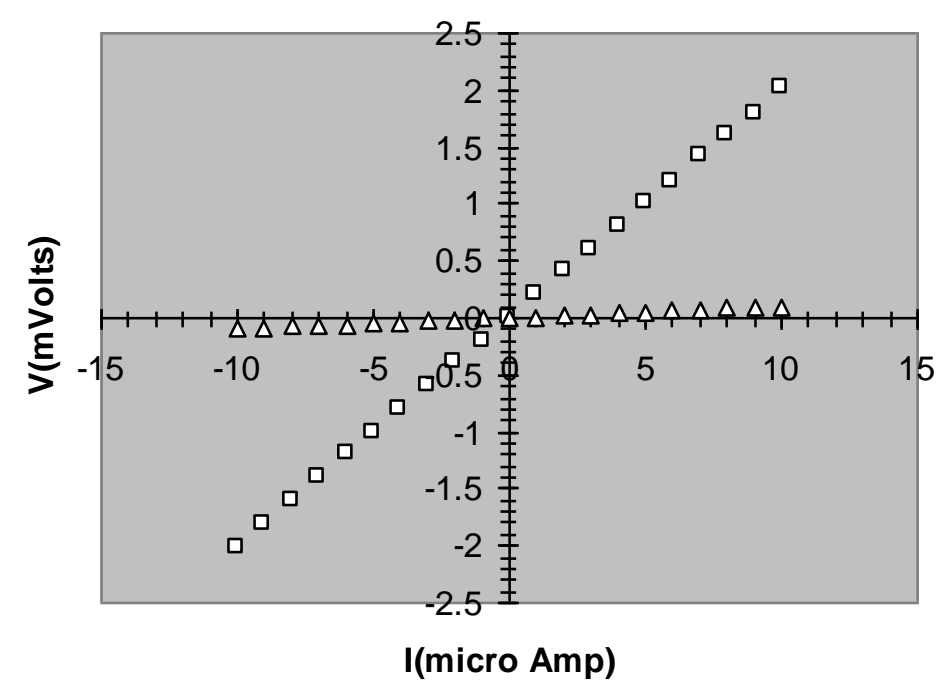

Figure 3. Voltage versus Current relationship for a typical sample in two perpendicular directions.

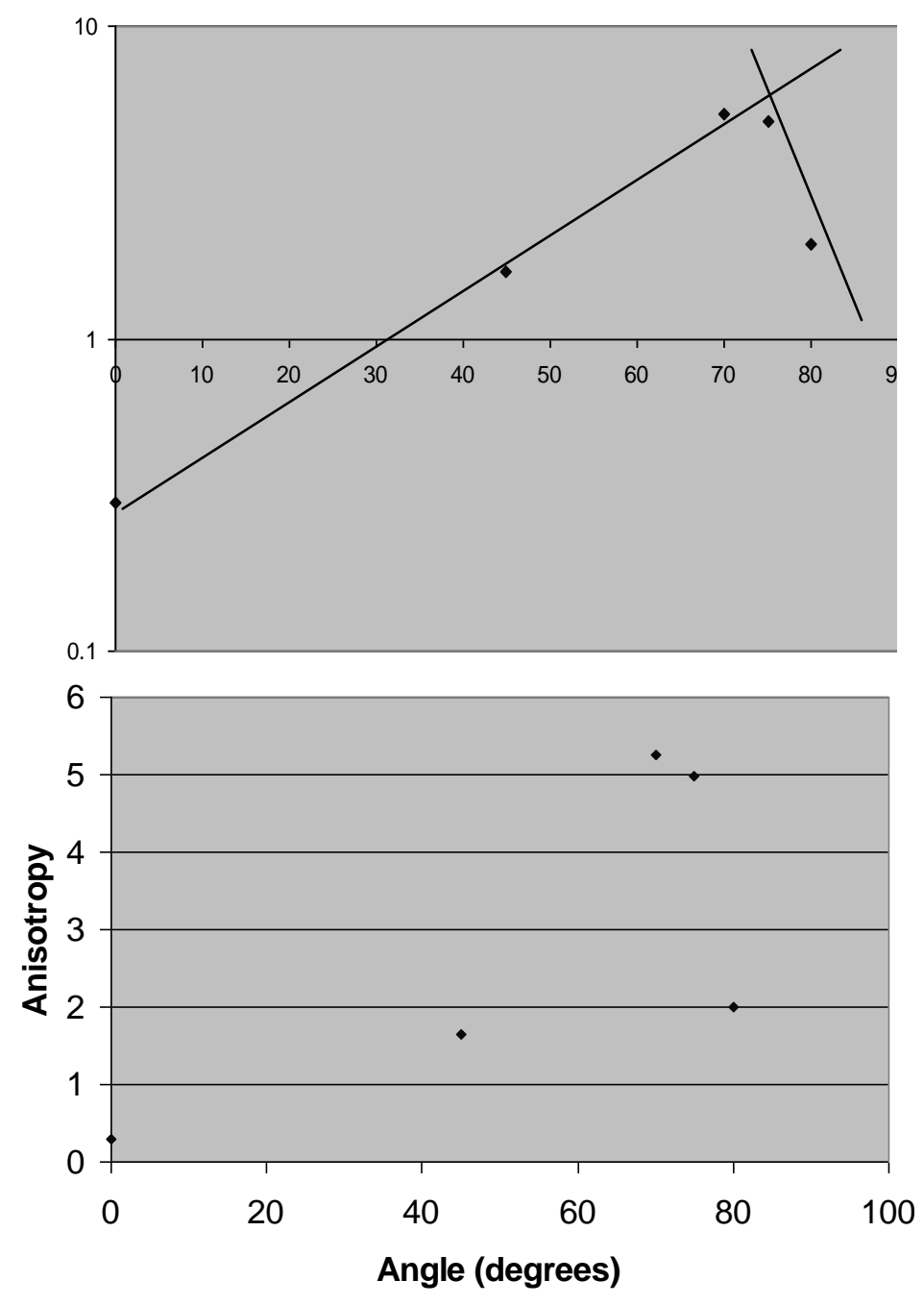

Figure 4. Resistivity anisotropy (the ratio $\rho_{x} / \rho_{y}$ ) versus angle $\theta$ : Linear scale (bottom); Semi log scale (top). Solid lines are extrapolations of the data. 
angle.

The exponential dependence of resistivity anisotropy on columns growth angle (and hence the inter columnar distance) suggests that the electrical conduction in the directions parallel to the surface of STFs may be as a result of quantum tunneling of the charged carriers [12]. Further investigations are underway to address this question in details.

\section{CONCLUSION}

The dependence of resistivity anisotropy of Chromium STFs on columns growth angle can qualitatively be explained by the structural inhomogenieties of the films. We have demonstrated that the electrical properties of the STFs can be manipulated by varying the column's growth angle.

\section{ACKNOWLEDGEMENTS}

The authors are in debt to Dr. Mark Horn, Department of Engineering Sciences and Mechanical Engineering, Pennsylvania State University, for providing us the samples and for his continuous support. We thank Dr. Aklesh Lakhtakia for his expert comments and encouragement. We are also thankful to Mr. Matthew Snyder and Mr. John Schreck (undergraduate students) for their valuable contributions. The research was partially supported by the Faculty Grants Committee Award (Org. \# 21710).

\section{REFERENCES}

1. Akhlesh Lakhtakia and Russell Messier, Sculptured Thin Fils - Nanoengineered Morphology and Optics, (SPIE, Bellinghum, USA, 2005).

2. Mark W. Horn, Mathew D. Pickett, Russell Messier, and Akhlesh Lakhtakia, J. Vac. Soc. Technol. B 22(6), 3426 (2004) and references therein.

3. A. Lakhtakia, Mater. Sci. Eng., C 19, 427 (2002).

4. K. Robbie, M. J. Brett, and A. Lakhtakia, J. Vac. Soc. Technol. A 13, 2991 (1995).

5. I. J. Hogkinson and Q. H. Wu, Adv. Mater. (Weinheim, Ger.) 13, 889 (2001).

6. M. Suzuki and Y. Taga, Jpn. J. Appl. Phys., Part 2 40, L358 (2001).

7. A. Lakhtakia, R. Messier, M. J. Brett, and K. Robbie, Innov. Mater. Res, 1(2), 165 (1996).

8. Akhlesh Lakhtakia, Microwave and Optical Tech. Lett., 2(4), 286 (1999).

9. Department of Engineering Sciences and Mechanical Engineering, Penn State University, University Park, PA16802.

10. H. C. Montgomery, J. App. Phys. 42(7), 2971 (1971).

11. I. J. Hogkinson and Q. H. Wu, Birefringent Thin Films and Polaring Elements, (World Scientific, Singapore, 1997).

12. Jianping Lin and David N. Beratan, J. Phys. Chem. A 108(26), 5655 (2004).

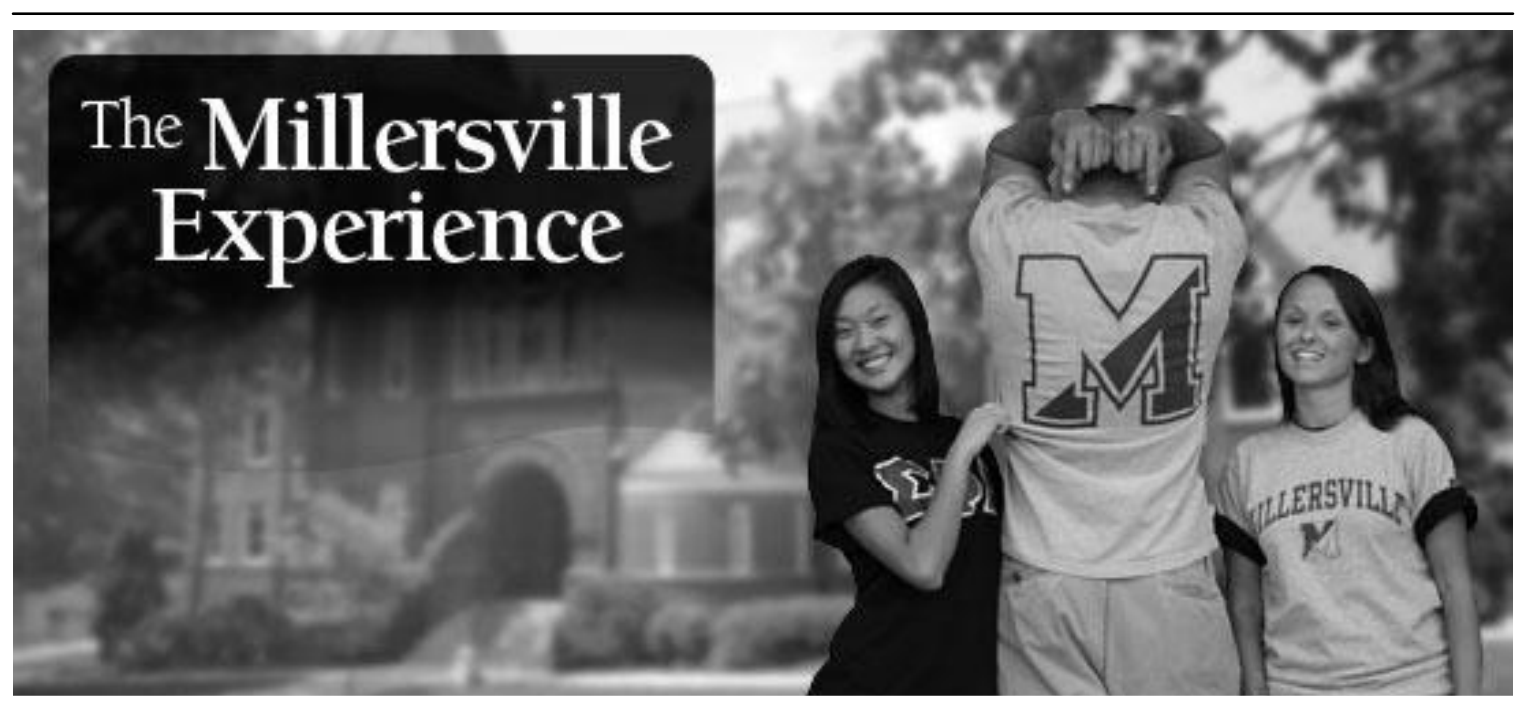

Millersville University $•$ Millersville, Pennsylvania, USA • www.millersville.edu 